\title{
3D intensity and phase imaging from light field measurements in an LED array microscope: supplementary material
}

\author{
LeI Tian* and LaURA WALLeR \\ Department of Electrical Engineering and Computer Sciences, University of California, Berkeley \\ *Corresponding author: lei_tian@alum.mit.edu
}

Published 28 January 2015

\begin{abstract}
This document provides supplementary information for "3D intensity and phase imaging from light field measurements in an LED array microscope," http://dx.doi.org/10.1364/optica.2.000104.

(C) 2015 Optical Society of America

http://dx.doi.org/10.1364/optica.2.000104.s001
\end{abstract}

\section{AXIAL RESOLUTION BASED ON 3D COHERENT TRANSFER FUNCTION ANALYSIS}

The lateral resolution of our multi-slice 3D Fourier Ptychography method is set by the sum of the objective NA $\left(\mathrm{NA}_{\mathrm{obj}}\right)$ and illumination NA ( $\left.\mathrm{NA}_{\text {illum }}\right)$, so will be equivalent to the situation of using a traditional imaging system with $\mathrm{NA}=\mathrm{NA}_{\mathrm{obj}}+\mathrm{NA}_{\text {illum }}$.

The axial resolution of our method, however, is predicted by the 3D coherent transfer function (CTF) analysis to be better than that of the original objective, but worse than the situation of a traditional imaging system with $\mathrm{NA}=\mathrm{NA}_{\mathrm{obj}}+\mathrm{NA}_{\text {illum }}$. We derive the axial bandwidth, $\Delta u_{z}$, as the maximum range of $u_{z}$ covered by the full set of measurements from all LEDs. Referring to the geometry in Fig. 6 of the main text, it can be written as

$$
\Delta u_{z}=\left(2-\sqrt{1-\mathrm{NA}_{\mathrm{obj}}^{2}}-\sqrt{1-\mathrm{NA}_{\mathrm{illum}}^{2}}\right) / \lambda,
$$

corresponding to an axial resolution of

$$
\delta_{z}^{\mathrm{fp}}=\lambda /\left(2-\sqrt{1-\mathrm{NA}_{\mathrm{obj}}^{2}}-\sqrt{1-\mathrm{NA}_{\mathrm{illum}}^{2}}\right) .
$$

For comparison, the axial resolution that would be achieved with an objective having $\mathrm{NA}=\mathrm{NA}_{\mathrm{obj}}+\mathrm{NA}_{\text {illum }}$ is

$$
\delta_{z}^{\mathrm{obj}}=\lambda /\left(1-\sqrt{1-\left(\mathrm{NA}_{\mathrm{obj}}+\mathrm{NA}_{\mathrm{illum}}\right)^{2}}\right) .
$$

Thus, the axial resolution in the $z$ dimension is not improved as much as lateral resolution when using the multi-slice Fourier Ptychography method, except in the limiting case. To give a sense of the trends in axial resolution for different situations, we plot in Fig. S1 both $\delta_{z}^{\mathrm{fp}}$ and $\delta_{z}^{\mathrm{obj}}$ for three typical objective NAs under various illumination NAs. In general, as illumination NA increases, the axial resolution differences reduce.

\section{NUMERICAL SIMULATIONS}

To study the axial sectioning capability of our technique, we simulate a test sample consisting of 4 axial slices with both amplitude and phase. The spacing between neighboring slices, $\Delta z$, is progressively decreased, beyond the predicted best axial resolution, in order to show how errors manifest. Simulated intensity images from each illumination angle are computed based on our multi-slice forward model (described in Section 2.B of the main text) with the simulated test object. The illumination and imaging parameters are chosen to match the experiments in Section 3 of the main text. Once the simulated dataset is created, it is then used as input to our reconstruction algorithm in order to solve for the phase and amplitude at each slice.

We first simulate the case of our $10 \times$ objective $(0.25 \mathrm{NA})$ using our multi-slice Fourier Ptychography algorithm, shown in Fig. S2. The axial resolution from the objective alone is $20 \mu \mathrm{m}$. By combining images from 225 illumination angles using multi-slice Fourier Ptychography, the axial resolution is improved to $5 \mu \mathrm{m}$ with LED illumination up to $\mathrm{NA}_{\text {illum }}=0.41$. As the slice spacing drops below this theoretical limit to $3 \mu \mathrm{m}$, prominent artifacts appear and the image fidelity drops significantly. Since the 3D CTF theory is based upon a single scattering approximation, the predicted axial resolution may become worse when considering multiple scattering, which is the case we study here. Our multi-slice model takes multiple scattering into account, which can only be partially mitigated in our reconstruction algorithm. Thus, even at a slice spacing of $10 \mu \mathrm{m}$, some artifacts begin to appear, though the slices are still resolvable. A similar study for our $4 \times$ objective ( $0.1 \mathrm{NA}$ ) with 0.41 illumination NA is also shown is in Fig. S3 to have similar trends. 

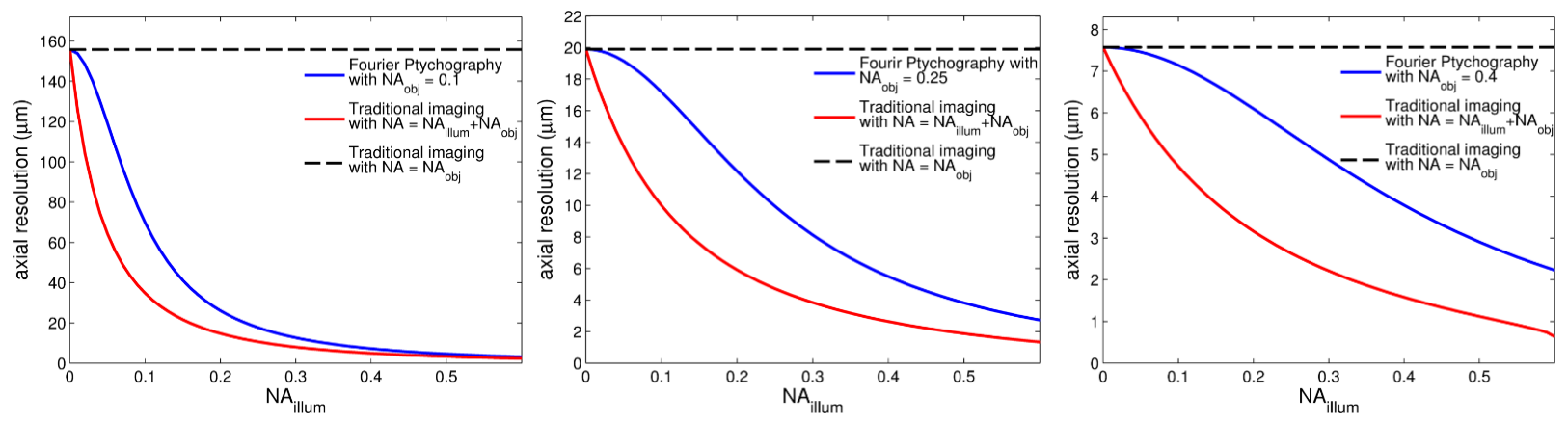

Fig. S1. The 3D CTF predicted axial resolution of multi-slice Fourier Ptychography (blue solid curves) for objectives with (a) $\mathrm{NA}_{\mathrm{obj}}=0.1$, (b) $\mathrm{NA}_{\mathrm{obj}}=0.25$, and (c) $\mathrm{NA}_{\mathrm{obj}}=0.4$, under various illumination NAs $\left(\mathrm{NA}_{\text {illum }}\right)$. The axial resolution from the native objective with $\mathrm{NA}=\mathrm{NA}_{\mathrm{obj}}$ (black dotted lines) and the high NA objective (red solid curves) having $\mathrm{NA}=\mathrm{NA}_{\mathrm{obj}}+\mathrm{NA}_{\mathrm{illum}}$ are plotted for comparison. 

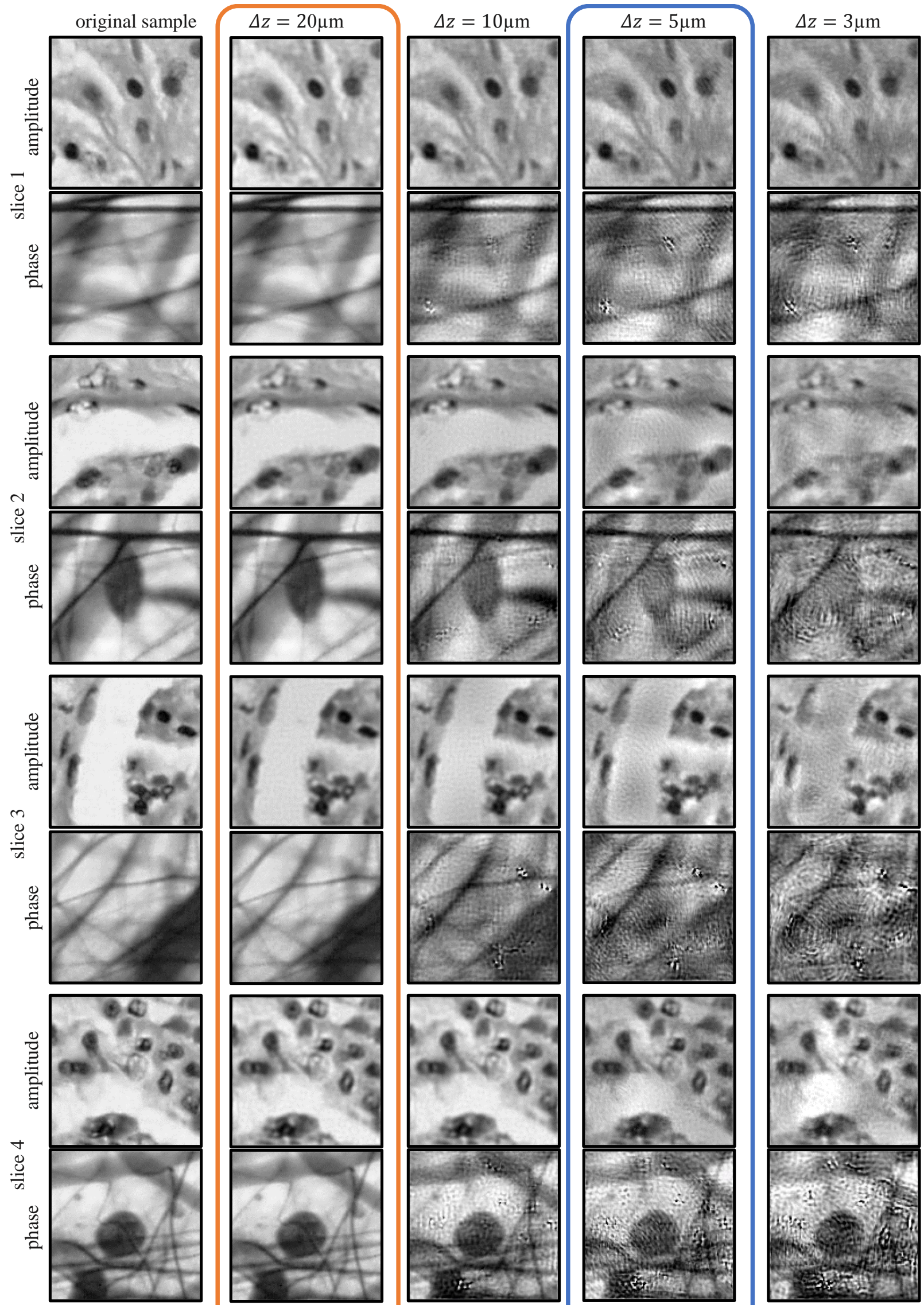

native objective

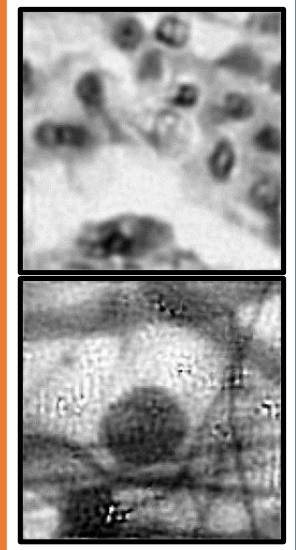

axial resolution

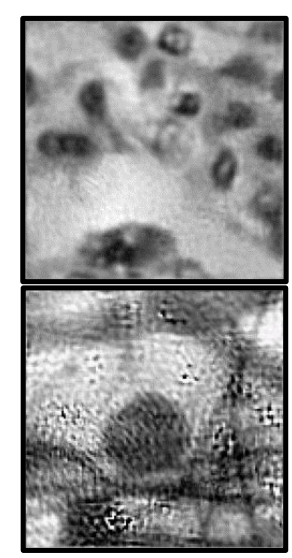

Fourier Ptychography
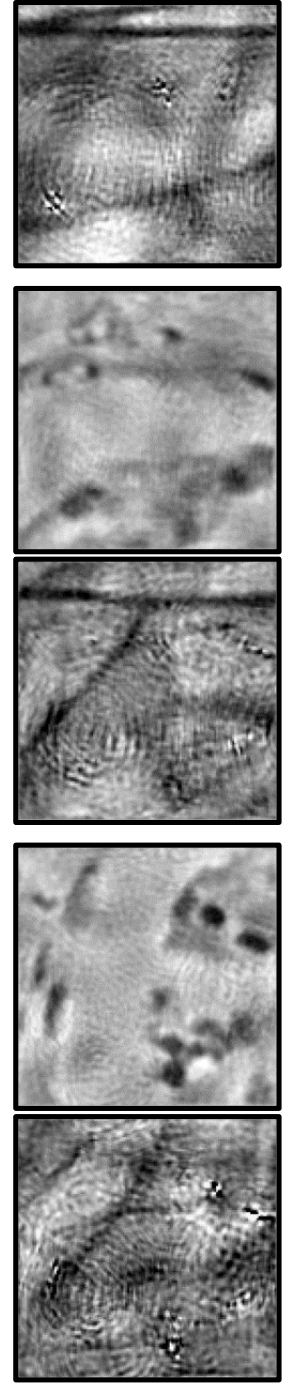

axial resolution

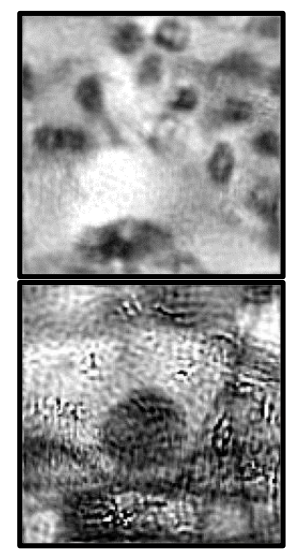

Fig. S2. Multi-slice Fourier Ptychography simulation results for $10 \times$ objective $(0.25 \mathrm{NA})$ with 0.41 illumination NA. The native objective axial resolution is $20 \mu \mathrm{m}$, and the 3D CTF predicts an axial resolution of $5 \mu \mathrm{m}$ by using Fourier Ptychography. At $3 \mu \mathrm{m}$ spacing, the slices are separated by less than the theoretical resolution limit, so suffer severe artifacts. 

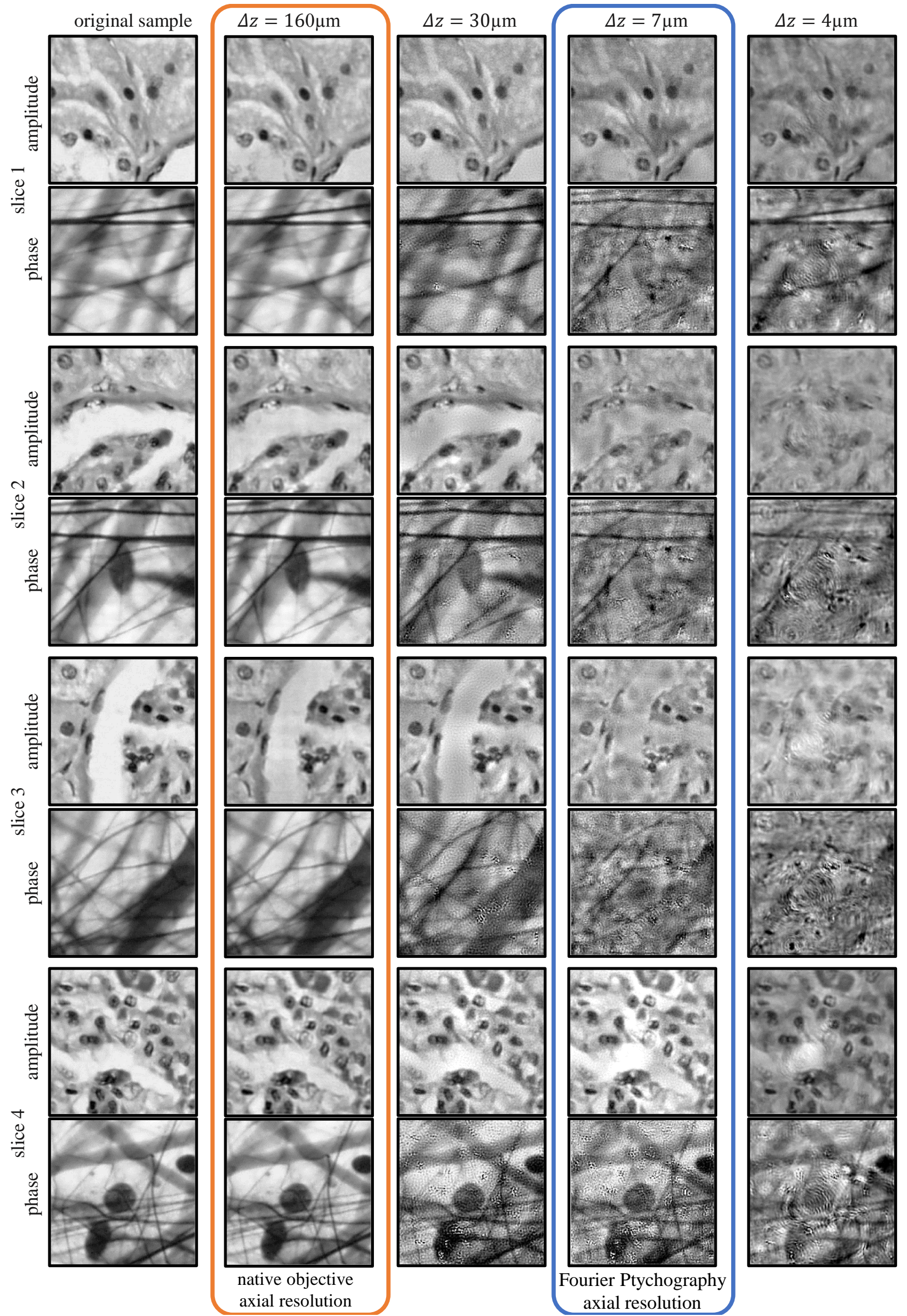

Fig. S3. Multi-slice Fourier Ptychography simulation results for $4 \times$ objective ( 0.1 NA) with 0.41 illumination NA. The native objective axial resolution is $160 \mu \mathrm{m}$, and the 3D CTF predicts an axial resolution of $7 \mu \mathrm{m}$ by using Fourier Ptychography. 